PD-1, CCR7, CXCR5, CD45RA antibodies. All patients fulfilled the ACR 1982 classification criteria for SLE. Cellular changes were analyzed in the context of clinical information.

Results: in the present cohort, the SLE patients were mainly female (86.6\%) and of median age of 36.7 (29.8-49.4) with a disease duration of 6.1(1.6-11.8) years, and active disease with SLEDAI-2K at baseline 12.0 (8.0-16.0). The frequency of age-associated $B$ cells (ABCs; CD27-IgD-CD11C+ CD21-) decreased by $13 \%$ $(p=0.03)$ in the first two to four months after rituximab start, while globally the DN (IgD-CD27-) B cells transiently increased by around $3 \%(p=0.15)$ at the first follow-up. This increase could not be attributed to the DN1 (CXCR5+CD11c-) or DN2 (CXCR5-CD11c+) subsets but to the CD11c-CXCR5- DN (DN3) B cells (increase $=6.7 \%, p=0.03$ ). In parallel, $T$ effector cells (CCR7- CD45RA+) and TEMRA (CD45RA+ CCR7-) frequencies increased after first follow up in both CD4+ and CD8+ $T$ cells. The frequency of $T_{F H}$ (CXCR5+ PD-1+) cells did not change after rituximab, however a decrease of PD- $1^{\text {high }} \mathrm{CD} 4+$ cells was observed in most patients, although not significant, after 2-4 month of treatment. In most patients the frequency of PD- $1^{\text {high }} \mathrm{CD} 4+$ cells either reduce or stay the same after RTX treatment (reduction $=0.53, p=0.28$ ). After $11-15$ months of RTX treatment the frequency of PD- $1^{\text {high }} \mathrm{CD} 4+\mathrm{T}$ cell reduces by a $-0.5 \%$ in comparison to $2-4$ months $(p=0.039)$. The SLEDAI at baseline did not correlate with the frequency of PD- $1^{\text {high }}$ CD4+ T cells $(r=0.03, p=0.9)$.

Conclusion: the importance of $\mathrm{T}$ cell - B cell interactions in SLE pathogenesis was recently strengthened by the identification of the lymphocyte subsets $T_{F H} /$ $T_{P H}$ and $A B C s$ respectively. Here, in the context of rituximab treated SLE, we could detect a reduction in the frequencies of both $A B C s$ and $P D-1^{\text {high }} T$ cells after treatment with rituximab, while the DN3 and effector memory $T$ cells frequencies increased. Our data suggests that anti-CD20 mediated B-cell depletion affects both B-cell and T-cell subsets frequencies, and that monitoring these specific cell subsets may be clinically relevant.

REFERENCES:

[1] Bocharnikov AV, Keegan J, Wacleche VS, Cao Y, Fonseka CY, Wang G, et al.

PD-1hiCXCR5- T peripheral helper cells promote $B$ cell responses in lupus via MAF and IL-21. JCI insight. 2019;4(20)

Disclosure of Interests: Francesca Faustini Speakers bureau: More than two years ago and not in relation to any aspect of the present research, Natalie Sippl: None declared, Ragnhild Stålesen: None declared, Karine Chemin: None declared, Iva Gunnarsson: None declared, Vivianne Malmström: None declared. DOI: 10.1136/annrheumdis-2021-eular.2366

\section{POS0004 SPONDYLOARTHROPATHIES EXHIBITS HALLMARKS OF HLA-DEPENDENT CLONAL EXPANSIONS AND REMAINS STABLE OVER 1.5 YEARS}

E. Komech ${ }^{1}$, A. Koltakova ${ }^{2}$, E. Shmidt ${ }^{3}$, A. Barinova ${ }^{1,4}$, M. Salnikova ${ }^{1}$ A. Minervina ${ }^{1}$, T. Korotaeva ${ }^{2}$, Y. Lebedev ${ }^{1}$, I. Zvyagin ${ }^{1,4}$. ${ }^{1}$ ShemaykinOvchinnikov Institute of Bioorganic Chemistry, Laboratory of Comparative and Functional Genomics, Moscow, Russian Federation; ${ }^{2}$ Nasonova Research Institute of Rheumatology, Lab of Spondyloarthritis and Psoriatic Arthritis, Moscow, Russian Federation; ${ }^{3}$ Pirogov Russian National Research Medical University, City Clinical Hospital \#1, Moscow, Russian Federation; ${ }^{4}$ Pirogov Russian National Research Medical University, Institute of Translational Medicine, Moscow, Russian Federation

Background: Different studies show involvement of T-cells in pathogenesis of spondyloarthropathies (SpAs) - a group of rheumatic diseases strongly associated with presence of several $\mathrm{MHC}-\mathrm{I}$ alleles (HLA-B*27, $-\mathrm{B}^{\star} 38,-\mathrm{B}^{\star} 39$, etc). Recently we and others identified a specific T-cell receptor motif in blood and synovial fluid of HLA-B*27+ AS patients that reinforces the "arthritogenic peptide" hypothesis of AS pathogenesis [1-2]. However, common characteristics of clonal T-cell repertoire of synovial fluid in SpAs remain poorly investigated.

Objectives: We aimed to investigate synovial fluid T-cell repertoires of SpA patients with different HLA-genotypes and stability of the clonal composition in recurring flares of the disease.

Methods: Mononuclear cells were isolated from paired peripheral blood (PB) and synovial fluid (SF) samples of SpA patients (ankylosing spondylitis and psoriatic arthritis, $n=27$ ). For 3 patients additional $S F$ samples were collected during relapse of the synovitis (after 9-15 months). CD4 and CD8 T-cells were isolated by immunomagnetic separation. Deep sequencing of UMl-tagged TCR beta cDNA libraries was used to accurately reconstruct clonal T-cell repertoires. HLA class I and II were typed for each donor using an in-house NGS-based system. Results: We observed restricted T-cell clonal composition in synovial fluid: on average only $6 \%$ of PB T-cell clonotypes were detected in SF of the same donor. T-cell repertoires of both CD4 and CD8 SF subsets compared to PB were highly oligoclonal (index Gini PB vs SF: CD4 $0.36 \pm 0.10$ vs $0.68 \pm 0.08$, CD8 $0.57 \pm 0.17$ vs $0.81 \pm 0.12$ ) in all patients. Number of identical amino acid CDR3 sequences between two repertoires correlated with the number of identical HLA-alleles for the donors. This trend was exhibited more strikingly in SF compared to PB, suggesting that common antigens may play a role in accumulation of identical T-cell clonotypes in the inflamed joint. Using several bioinformatic approaches we identified groups of highly similar SF clonotypes linked to HLA-B*27 and/or HLA-B ${ }^{\star} 38$ genotype.

Total SF repertoires of relapsing synovitis of the same donor showed huge clonal overlap, and the most frequent clonotypes remained almost unchanged (Morisita's overlap index for total SF repertoires $0.69 \pm 0.26$; for top 1000 clonotypes $0.79 \pm 0.19, n=3$ ).

Conclusion: We report HLA-dependent sharing of identical and similar T-cell clonotypes in SF of patients with ankylosing spondylitis and psoriatic arthritis and high stability of SF repertoire during several flares that support antigen-driven accumulation of T-cells in the site of inflammation

\section{REFERENCES:}

[1] Komech EA et al. Rheumatology (Oxford). 2018;57(6):1097-1104.

[2] Faham M et al. Arthritis Rheumatol. 2016;11(10):300-308.

Acknowledgements: The work is supported by Russian Science Foundation grant №20-75-00041.

Disclosure of Interests: None declared.

DOI: 10.1136/annrheumdis-2021-eular.3498

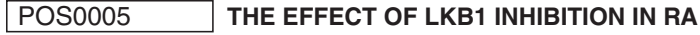 PATHOGENESIS}

H. R. Lee ${ }^{1,2}$, J. Kim², S. J. Yoo ${ }^{2}$, J. A. Park ${ }^{2}$, S. W. Kang ${ }^{1,2} \cdot{ }^{1}$ Chungnam National University School of Medicine, Research Institute for Medical Sciences, Daejeon, Korea, Republic of (South Korea); ${ }^{2}$ Chungnam National University Hospital, Department of Internal Medicine, Daejeon, Korea, Republic of (South Korea)

Background: Liver kinase B1 (LKB1) is known as a tumor suppressor gene and also inhibits reactive oxygen species (ROS) levels. Intracellular ROS are catalyzed by the enzyme complex nicotinamide adenine dinucleotide phosphate (NADPH) oxidase (NOX). We previously reported that NOX4 induced the migration and invasion of fibroblast-like synoviocytes (FLS) in rheumatoid arthritis (RA). Although LKB1 is expected to alleviate synovial inflammation through ROS regulation, the role of LKB1 in RA has not been examined.

Objectives: To explore whether LKB1 affects RA inflammation, we transfected LKB1 siRNA and analyzed related gene expressions in RA FLS.

Methods: Synovial tissues were obtained from RA patients who were undergoing synovectomy or joint replacement. The isolated cells were cultured in Dulbecco's modified Eagle's medium (DMEM) supplemented with $10 \%$ fetal bovine serum, $100 \mathrm{U} / \mathrm{ml}$ penicillin and $100 \mathrm{mg} / \mathrm{ml}$ streptomycin and maintained in a $5 \%$ $\mathrm{CO}$ incubator at $37{ }^{\circ} \mathrm{C}$. FLS were used for experiments after four to six passages. Cells were transfected with lipofectamine transfection reagent and LKB1 siRNA duplex targeting constructs. After incubation for $24 \mathrm{~h}$, downregulation of target expression was evaluated by real-time PCR and western blot analysis.

Results: RA FLS was transfected with LKB1 siRNA and $90 \%$ of LKB1 mRNA expression was decreased. LKB1 knock-down also caused the decreased expression of mechanistic target of rapamycin (mTOR; 0.38 fold) and serine/threonine kinase (AKT) 2 ( 0.40 fold), which are downstream targets of LKB1. NOX4 was significantly increased (4.94 fold) by LKB1 inhibition. On the other side, the down regulated NOX4 induced significantly elevated LKB1 mRNA expression in RA FLS. When the expressions of proinflammatory cytokines were examined, IL-1 $\beta$, IL-6, TNF- $\alpha$ were highly increased by LKB1 deficiency. FLS migration-related chemokines, IL-8 and MMP-3 were also enhanced compared to control. Conclusion: There was a negative correlation between NOX4 and LKB1 in RA FLS. As LKB1 deficiency induced the expression of proinflammatory cytokines and migration related chemokines, LKB1 could play a critical role in RA pathogenesis.

\section{REFERENCES:}

[1] Bartok B, Firestein GS. Fibroblast-like synoviocytes: key effector cells in rheumatoid arthritis. Immunol Rev. 2010;233(1):233-55.

[2] Mateen S, Moin S, Khan AQ, Zafar A, Fatima N. Increased reactive oxygen species formation and oxidative stress in rheumatoid arthritis. PLoS One. 2016;11(4):e0152925.

Disclosure of Interests: None declared.

DOI: 10.1136/annrheumdis-2021-eular.946

\section{POS0006 \\ NX-5948, A SELECTIVE DEGRADER OF BTK, SIGNIFICANTLY REDUCES INFLAMMATION IN A MODEL OF AUTOIMMUNE DISEASE}

D. Robbins ${ }^{1}$, M. Noviski ${ }^{2}$, M. Tan ${ }^{3}$, C. Guiducci ${ }^{2}$, T. Ingallinera ${ }^{4}$, D. Karr ${ }^{4}$, A. Kelly ${ }^{2}$, Z. Konst ${ }^{1}$, A. Tenn-Mcclellan ${ }^{2}$, J. Mckinnell ${ }^{4}$, L. Perez ${ }^{2}$, G. Hansen ${ }^{5}$, R. Rountree ${ }^{2} .{ }^{1}$ Nurix Therapeutics, Medicinal Chemistry, San Francisco, United States of America; ${ }^{2}$ Nurix Therapeutics, Discovery Biology, San Francisco, United States of America; ${ }^{3}$ Nurix Therapeutics, Translational Medicine, San Francisco, United States of America; ${ }^{4}$ Nurix Therapeutics, Preclinical 